\title{
Regulation of plant immune receptors by ubiquitination
}

\section{Giulia Furlan $^{\dagger}$, Jörn Klinkenberg ${ }^{\dagger}$ and Marco Trujillo*}

Leibniz Institute of Plant Biochemistry, Halle (Saale), Germany

\section{Edited by:}

Jacqueline Monaghan, The Sainsbury

Laboratory, UK

\section{Reviewed by:}

Zhi-Yong Wang, Carnegie Institution for Science, USA

Sandra Goritschnig, University of

California at Berkeley, USA

\section{*Correspondence:}

Marco Trujillo, Leibniz Institute of Plant Biochemistry, Weinberg 3, D-06120 Halle (Saale), Germany.

e-mail: mtrujillo@ipb-halle.de

${ }^{\dagger}$ Giulia Furlan and Jörn Klinkenberg have contributed equally to this work.
From pathogen perception and the activation of signal transduction cascades to the deployment of defense responses, protein ubiquitination plays a key role in the modulation of plant immunity. Ubiquitination is mediated by three enzymes, of which the E3 ubiquitin ligases, the substrate determinants, have been the major focus of attention. Accumulating evidence suggests that ubiquitination modulates signaling mediated by pattern recognition receptors and is important for the accumulation of nucleotide-binding leucine-rich repeat type intracellular immune sensors. Recent studies also indicate that ubiquitination directs vesicle trafficking, a function that has been clearly established for immune signaling in animals. In this mini review, we discuss these and other recent advances and highlight important open questions.

Keywords: E3 ubiquitin ligases, vesicle trafficking, receptor-like kinases, effectors, protein degradation, ubiquitination, PTI

\section{INTRODUCTION}

Ubiquitin is a highly conserved protein found in all eukaryotes and is involved in almost all aspects of plant physiology, including immunity. Ubiquitination is the reversible attachment of ubiquitin moieties to specific target proteins and it is mediated by three enzymes (Vierstra, 2009). In the initial step, ubiquitin is activated by an ubiquitin-activating enzyme (E1). The activated ubiquitin is then transferred to an E2 ubiquitin-conjugating enzyme. A ubiquitin ligase (E3) then binds the E2 and the target protein. The ligase generally acts as a scaffold bringing the $\mathrm{E} 2$ and the target into close proximity to mediate the linkage of ubiquitin via its $\mathrm{C}$-terminal glycine to an $\varepsilon$-Lysine (Lys) residue of the target. Because E3 ligases determine the specificity of the reaction they have attracted by far the most attention. Target proteins can be modified by the attachment of single ubiquitin molecules (monoubiquitination) or of ubiquitin polymers linked internally through one of seven Lys residues present in ubiquitin (polyubiquitination). Generally, conjugated ubiquitin monomers or polymers act as portable recognition modules that facilitate protein-protein interaction.

Lys48-linked ubiquitin chains promote protein breakdown by the $26 \mathrm{~S}$ proteasome, a proteolytic complex that degrades the target with the concomitant release of the ubiquitin moieties for reuse. Alternatively linked ubiquitin chains can direct non-proteolytic events that participate in the regulation of vesicular trafficking, chromatin structure, and transcription (Ikeda and Dikic, 2008).

Post-translational modifications, such as ubiquitination, play key roles in signal transduction cascades. Understanding how such modifications translate into signal modulation has become a major research focus in recent years. This mini review focuses on recent reports implicating ubiquitination in the regulation of plant immune sensors and vesicle trafficking.

\section{UBIQUITINATION AND PATHOGEN PERCEPTION}

The plant immune system can be conceptually divided into two branches characterized by different types of receptors (Jones and
Dangl, 2006). The first branch is mediated by plasma membrane (PM) located pattern recognition receptors (PRRs), which recognize conserved pathogen molecules, so called pathogenassociated molecular patterns (PAMPs). Recognition of PAMPs by PRRs ultimately results in PAMP-triggered immunity (PTI). The second branch is activated by intracellular nucleotide-binding leucine-rich repeat (NB-LRR) immune sensors, which directly or indirectly perceive virulence factors, known as effectors, and results in the activation of effector-triggered immunity (ETI; Bent and Mackey, 2007).

A connection between ubiquitination and plant immunity was first suggested by a study showing that suppressor of $G 2$ allele of skp 1 (sgt1) mutants were compromised in ETI (Azevedo et al., 2002). SGT1 is a component of the RAR1 (required for MLA12 resistance 1)-SGT1-HSP90 (heat shock protein 90) chaperone complex and association with components involved in protein ubiquitination has been shown for members of this complex. For example, S-phase kinase-associated protein 1 (SKP1) and its associated protein Cullin1, which are subunits of SKP1-Cullin1-F-box (SCF) ubiquitin ligases, were found to interact with SGT1 in plants (Azevedo et al., 2002; Liu et al., 2002). Subsequent studies have demonstrated that this chaperone complex plays a central role in the accumulation of NB-LRR proteins (Shirasu, 2009).

More recent findings have shown the direct regulation of NB-LRR accumulation through ubiquitin-mediated degradation via the $26 \mathrm{~S}$ proteasome. Loss-of-function mutation of constitutive expressor of PR genes 1 (CPR1, also named CPR30), which encodes an F-box motif protein, leads to the accumulation of the Toll-interleukin-receptor-like (TIR) type NB-LRR protein SNC1 (suppressor of npr1-1, constitutive 1) and the coiled-coil (CC) type NB-LRR protein RPS2 (resistance to Pseudomonas syringae 2) resulting in autoimmune responses (Cheng et al., 2011; Gou et al., 2012). Accordingly, CPR1 overexpression reduced SNC1 and RPS2 levels and immune response intensity. $\mathrm{CPR} 1$ was shown to interact with the ASK1 (Arabidopsis SKP1) and ASK2 subunits of SCF 
complexes. Furthermore, CPR1 interacts with SNC1 and RPS2, suggesting that they are its ubiquitination substrates and therefore mediate their stability (Gou et al., 2009; Cheng et al., 2011). Degradation mediated by CPR1 may reflect fine tuning mechanisms by which the plant is able to mount an immune response of appropriate intensity.

Another example of NB-LRR regulation by ubiquitination comes from a study conducted by Jeong et al. (2010), who uncovered an interesting link between light perception and immunity mediated by hypersensitive response to TCV (HRT), a CC-NBLRR which mediates resistance against the turnip crinkle virus (TCV). HRT protein levels decreased in the dark or after bluelight induction, resulting in enhanced susceptibility. Application of proteasome inhibitor prevented blue-light-dependent degradation of HRT and consequently, plants were more resistant to TCV (Cooley et al., 2000; Jeong et al., 2010). HRT accumulation was reduced in mutants of the blue-light receptors cryptochrome 2 (CRY2) and phototropin 2 (PHO2). Importantly, HRT interacted with the ubiquitin ligase constitutively photomorphogenic 1 (COP1), but not with CRY2 or PHO2 (Jeong et al., 2010). Because $\mathrm{CRY} 2$ and $\mathrm{PHO} 2$ do interact with COP1 and they are required for HRT accumulation, it was proposed that they negatively regulate HRT degradation via COP1. However, the exact function of COP1 still remains to be determined.
In contrast to the intracellular NB-LRR immune sensors, surface-localized PRR receptor-like kinases (RLKs), relay external cues into the cell. PRRs recognize PAMPs such as flagellin, a component of the bacterial flagella, or chitin, a component of the fungal cell wall (Monaghan and Zipfel, 2012). Indication for the involvement of ubiquitination in the regulation of PRR signaling was first provided by the bacterial effector protein AvrPtoB, which is an active E3 ligase with a C-terminal U-box/RINGlike domain (Janjusevic et al., 2006). AvrPtoB physically interacts with and ubiquitinates the flagellin receptor flagellin-sensitive 2 (FLS2; Figure 1A). Expression of AvrPtoB resulted in a reduction of FLS2 levels, indicating that AvrPtoB facilitates its degradation (Göhre etal., 2008). AvrPtoB is also able to ubiquitinate and mediate the degradation of at least one more PRR, namely the chitin receptor chitin elicitor receptor kinase 1 (CERK1; Gimenez-Ibanez et al., 2009).

Recently, the plant U-box (PUB) ligases PUB12 and PUB13 were shown to mediate the endogenous ubiquitination of FLS2 (Lu et al., 2011; Figure 1B). PUB12 and PUB13 interact constitutively with brassinosteroid-insensitive 1-associated receptor kinase 1 (BAK1), while treatment with flg22, a conserved N-terminal peptide of flagellin, is required to induce their interaction with FLS2 (Chinchilla et al., 2007). Although phosphorylation of PUB12 and PUB13 by BAK1 was needed for the interaction with FLS2, it

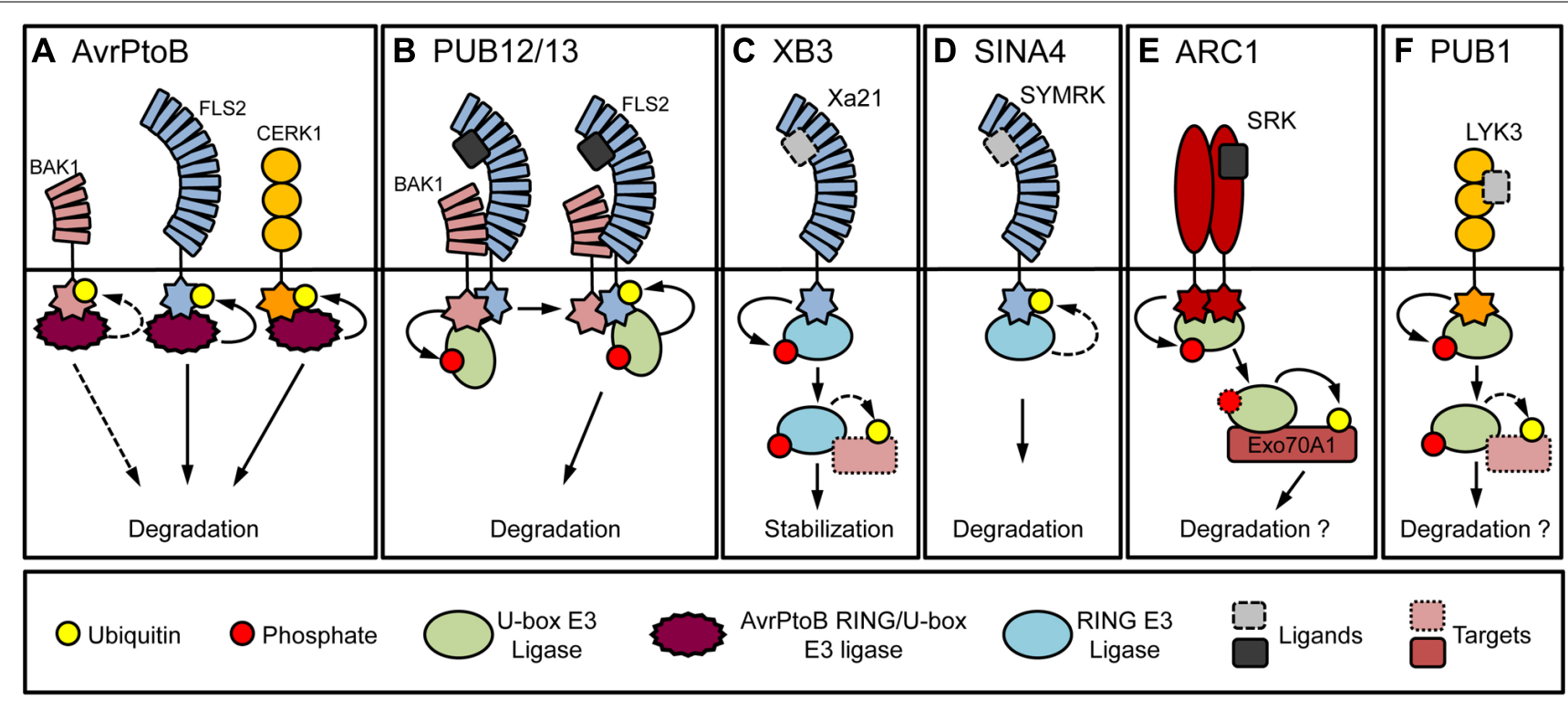

FIGURE 1 | Ubiquitin ligases that interact with receptor kinases. (A) The effector protein AvrPtoB from P. syringae pv. tomato binds to the co-receptor BAK1, the LRR-RLK FLS2 and the LysM RLK CERK1. AvrPtoB is able to ubiquitinate FLS2 and CERK1 and mediate their degradation. AvrPtoB can ubiquitinate BAK1 weakly in vitro. The mechanism leading to reduced RLK levels by AvrPtoB activity in vivo requires further clarification. (B) PUB12 and PUB13 constitutively interact with the co-receptor kinase BAK1. Constitutive phosphorylation of PUB12 and PUB13 by BAK1 is enhanced by flg22 which induces the interaction with FLS2. PUB12 and PUB13 ubiquitinate FLS2 and mediate its degradation. (C) The rice XB3 ligase interacts with the LRR-RLK XA21. XA21 phosphorylates XB3 in vitro. Whether ligand binding is required for the phosphorylation is not known. XB3 contributes to XA21 accumulation and is therefore unlikely to ubiquitinate XA21. XB3 conceivably targets a protein that affects XA21 accumulation. (D) The L. japonicus SINA4 was shown to interact with and negatively regulate the levels of the LRR-RLK SYMRK, which mediates symbiotic signaling. (E) The B. napus ARC1 interacts and is phosphorylated by the S-domain SRK, which mediates SI reaction. ARC1 was proposed to regulate SI through the degradation of Exo70A1. Further experimental clarification is needed to determine whether ARC1 affects SRK levels. (F) The $M$. truncatula PUB1 interacts with and is phosphorylated by LYK3, a LysM type RLK involved in nodulation. PUB1, a negative regulator of nodulation, does not ubiquitinate LYK3 in vitro. PUB1 might therefore target an alternate protein required for symbiosis. Shapes with dotted lines denote potential involvement (e.g., ligand) or a hypothetical target. 
was dispensable for FLS2 ubiquitination. Interestingly, functional analysis of pub12 and pub13 mutants showed a phenotype reminiscent of pub22, pub23, and pub24 mutants, which included enhanced responses to PAMPs and resistance to pathogens (Trujillo et al., 2008; Lu et al., 2011). Importantly, pub12/pub13 double mutants displayed impaired reduction of FLS2 protein levels after flg22 treatment, indicating that they participate in the attenuation of signaling by regulating FLS2 turn-over. Of note, neither PUB12 or PUB13, nor AvrPtoB are able to effectively ubiquitinate BAK1 in vitro or affect BAK1 levels in vivo. Also, in vitro ubiquitination of FLS2 by PUB12, PUB13, and AvrPtoB is independent of its putative PEST domain although its mutation impairs endocytosis (Robatzek et al., 2006; Göhre et al., 2008; Lu et al., 2011). This suggests that FLS2 endocytosis and PUB12, PUB13, and AvrPtoB mediated degradation could be uncoupled.

PUB13 may have additional functions as suggested by studies which show that it negatively regulates cell death and influences flowering time (Li et al., 2012). pub13 plants showed enhanced resistance against hemibiotrophic bacterial pathogens, in line with the results shown by Lu et al. (2011). Additionally, pub13 mutants also displayed enhanced susceptibility to a necrotrophic pathogen (Li et al., 2012). Similarly, mutants of a gene encoding the putative PUB13 ortholog in rice, spotted leaf 11 (SPL11), were also reported to show spontaneous cell death and altered defense responses (Zeng et al., 2004). Interestingly, both orthologs additionally affect flowering time regulation, although they display opposing phenotypes. Whereas flowering starts earlier in pub13 plants, it is delayed in rice spl11 mutants grown under long day conditions (Vega-Sanchez et al., 2008; Li et al., 2012). Both resistance and flowering time phenotypes were shown to be largely dependent on constitutively increased SA levels in pub13, as introgression of phytoalexin deficient 4 (pad4) or salicylic acid induction deficient 2 (sid2) mutations suppressed both phenotypes.

In rice, the ubiquitin ligase XB3 (XA21-binding protein 3) interacts in vivo with the PRR XA21 (Xanthomonas oryzae pv. oryzae resistance 21 ), which is also able to phosphorylate $\mathrm{XB} 3$ (Wang et al., 2006). Reduced expression of XB3 results in lower protein levels of XA21 and decreased resistance to the avirulent $X$. oryzae pv. oryzae, suggesting that XB3 is required for the accumulation of XA21 (Figure 1C). In Lotus japonicus, the RING-type ligase seven in absentia 4 (SINA4), was shown to interact with symbiosis RLK (SYMRK) and to negatively influence infection thread development during rhizobia infection (Den Herder et al., 2012; Figure 1D). Expression of SINA4 reduced SYMRK levels indicating a regulatory function of SINA4.

Because PRRs are integral membrane proteins, regulation of protein levels requires different cellular processes than in the case of NB-LRRs. Transport of RLKs to and from the PM is mediated by vesicle trafficking. Ubiquitination is closely involved in many steps of vesicle trafficking; it directs trafficking decisions related to both the biosynthetic secretory pathway and the removal of PM proteins via the endocytic pathway. Cell signaling and endocytic trafficking of membrane proteins have traditionally been regarded as two independent processes. However, recent studies, mainly from nonplant systems, have demonstrated that these two processes are intimately intertwined (Scita and Di Fiore, 2010).

\section{UBIQUITINATION AND IMMUNE RECEPTOR TRAFFICKING}

Remodeling of the PM protein composition is emerging as a key aspect regulating receptor signaling and mediating signal resolution in space and time (Scita and Di Fiore, 2010). Endocytosis can regulate cell signaling by controlling the number of available receptors. This paradigm has been demonstrated for several receptors in animal cells including receptor tyrosine kinases, G protein-coupled receptors, and others (for review, see Sorkin and von Zastrow, 2009).

Recent studies suggest that a similar paradigm could be valid in plants. The receptor FLS2 is internalized and degraded in response to binding to flg22 (Robatzek et al., 2006). Internalization and concomitant degradation have been suggested to mediate signal attenuation.

The mechanism by which AvrPtoB-, PUB12-, or PUB13mediated PRR ubiquitination modulates protein levels, still remains to be clarified. PRR ubiquitination can lead to one of many fates which can include endocytosis, changes in PRR sorting after endocytosis or in protein secretion. In humans, toll-like receptor (TLR)-mediated signaling is regulated by the RINGtype ligase Triad3A. Both the TLR4 and TLR9 are ubiquitinated by Triad3A leading to their degradation upon activation with lipopolysaccharide (LPS) and cytosine-guanosine dinucleotide motifs (CpG), respectively (Chuang and Ulevitch, 2004). However, initial endocytosis of the LPS receptor complex can also take place in a ubiquitination-independent manner (Husebye et al., 2006). This suggests that receptor ubiquitination may regulate protein levels by modulating PRR traffic at different stages after endocytosis.

Following internalization, cargoes go through a sorting process which decides whether they will be recycled and returned to the PM, or transported to the vacuole for degradation via multi-vesicular bodies (MVBs). This additional level of regulation is mediated by the endosomal sorting complex required for transport (ESCRT). Several studies showed that monoubiquitination of integral PM proteins is required for sorting into MVBs in yeast and animal cells (Hicke, 2001; Haglund et al., 2003; Raiborg et al., 2003).

In plants, one of the first studies to show the involvement of ubiquitination in vacuolar sorting was provided by Kasai et al. (2011). They demonstrated that the substitution of the Lys590 residue, which is mono- or diubiquitinated in vivo, blocked the degradation of the borate transporter BOR1. Furthermore, the Lys590Ala mutation impaired translocation from the early endosome (EE) and transport to the vacuole without affecting localization to the PM. A recent study suggested a potential role of monoubiquitination in the degradation of the iron-regulated transporter 1 (IRT1), an integral PM protein, via the lytic vacuole (Barberon et al., 2011). IRT1 was shown to cycle between the PM, trans-Golgi network (TGN)/EE, and the vacuole to maintain optimal metal uptake. However, mutation of putative ubiquitin-conjugation residues led to IRT1 stabilization at the PM. In addition, artificial monoubiquitination of the PM ATPase was sufficient to cause its endocytosis and targeting to the vacuole, supporting monoubiquitination as signal for vacuolar targeting (Herberth etal., 2012). Because RLKs are integral membrane proteins, it is likely that they are also subject to similar 
processes in which ubiquitination orchestrates sorting into the vacuole.

Various components of the ESCRT bind ubiquitin and the deubiquitinating enzyme AMSH3 (associated molecule with the $\mathrm{SH} 3$ domain of STAM3) has been proposed to promote recycling of endocytosed proteins in animal cells. The Arabidopsis AMSH3 homolog is involved in vacuole biogenesis and vesicular traffic in general, including endocytosis (Isono et al., 2010). Interestingly, AMSH3 interacts with the ESCRT-III subunits vacuolar protein sorting 2.1 (VPS2.1) and VPS24.1 and regulates their localization (Katsiarimpa et al., 2011).

In the secretory pathway, components of the endoplasmic reticulum (ER)-quality control ensure the proper accumulation of PRRs. ER-quality control was shown to be required for the accumulation and proper function of elongation factor-Tu receptor (EFR) and FLS2 receptors (Nekrasov et al., 2009; Saijo et al., 2009). Mutant plants of the stromal-derived factor-2 (SDF2) and the luminal binding protein (BiP), a member of the Hsp70 family of chaperones, were impaired in PAMP-triggered responses and resistance against the pathogens $P$. syringae and Alternaria brassicicola (Nekrasov et al., 2009). The ER-quality control machinery is largely dependent on ubiquitination of defective proteins to mediate their degradation (Smith et al., 2011).

In addition, components of the ER-associated protein degradation (ERAD), such as the stress-induced ubiquitin-conjugating enzyme 32 (UBC32), participate in the secretion control of integral PM proteins. Transient expression of UBC32 in tobacco resulted in the reduced accumulation of the barley powdery mildew resistance locus O-12 (MLO12), a known substrate of ERAD (Müller et al., 2005; Cui et al., 2012). The bri1-9 and bri1-5 mutant alleles of the brassinosteroid-insensitive 1 (BRI1) receptor cause the ER-retention of the functional receptors and the typical brassinosteroid-insensitive dwarf phenotype (Jin et al., 2007; Hong etal., 2008). The double mutant ubc32/bri1-9 partially rescues the bri1-9 dwarf phenotype by allowing the functional bri1-9 mutant form to bypass ERAD and accumulate. Because UBC32 is induced by various ER stressors, it is conceivable that it participates in the regulation of ERAD stress responses (Cui et al., 2012). In line with these observations, two homologs of the ER membrane-localized RING-type ubiquitin ligase of the yeast and mammalian Hrd1, were shown to function redundantly in bri1-9 ERAD (Su etal., 2010). Double mutants of the two Arabidopsis Hrd1 homologs suppressed the bri1-9 phenotype. The former observations are also interesting in light of recent data that show the antagonism between brassinosteroid and immune signaling (Albrecht et al., 2011; Belkhadir et al., 2011).

\section{REGULATION OF UBIOUITIN LIGASES}

In many cases ubiquitin ligases are phosphorylated by interacting RLKs. It is therefore tempting to speculate that ligase phosphorylation regulates their activity or the interaction with target proteins.

One of the first examples showing such an interaction was the U-box type ubiquitin ligase from Brassica napus arm repeat containing 1 (ARC1) and S receptor kinase (SRK) which regulates self-incompatibility (SI; Figure 1E; Stone et al., 1999).
ARC1 was shown to be phosphorylated by SRK (Gu et al., 1998). Interestingly, phosphorylation was required for the relocalization of ARC1 from the cytosol to the ER (Stone et al., 2003). Yeast two-hybrid analysis with different S-domain RLKs and several Arabidopsis PUBs suggested the conservation of the SI signaling pathway in Arabidopsis (Samuel et al., 2008). The Arabidopsis Sdomain RLKs Arabidopsis receptor kinase 1 (ARK1) and ARK2 were also able to phosphorylate PUB9 and PUB13 in vitro. In addition, the Nicotiana benthamiana RLK CHRK1 had previously been reported to interact with NtPUB4, the homolog of BnARC1 (Kim et al., 2003).

The Medicago truncatula PUB1 was shown to interact with lysin motif RLK 3 (LYK3), a putative RLK of Sinorhizobium meliloti Nod factors (Mbengue et al., 2010). PUB1 was also phosphorylated by LYK3, but was unable to ubiquitinate it in vitro (Figure 1F). Overexpression and knock-down experiments suggested that PUB1 is a negative regulator of infection and nodulation by S. meliloti.

In the case of PUB12 and PUB13, BAK1-mediated phosphorylation induced their association with FLS2 (Lu etal., 2011), suggesting that phosphorylation modulates ligase affinity and thus mediates the association to FLS2. However, PUB12 and PUB13 phosphorylation does not seem to be required for target ubiquitination, since they readily ubiquitinated FLS2 in vitro. Furthermore, most RING and PUB ligases display in vitro autoubiquitination, suggesting that additional factors are dispensable for their activity. Nevertheless, it still remains unknown whether PUB1, SINA4, or XB3, as well as other mentioned ubiquitin ligases, can ubiquitinate the corresponding RLKs. Instead, it is conceivable that phosphorylation triggers the interaction with alternative targets.

The relocalization of proteins prompted by interaction with ubiquitin ligases is a reoccurring theme. Intracellular relocalization of ubiquitin ligases may represent a mechanism by which their activity is restricted to a specific cellular context. The RING-type ligase keep on going (KEG) functions in abscisic acid signaling and its mutation suppresses the enhanced resistance against powdery mildew in enhanced disease resistance 1 (edr1) plants (Wawrzynska et al., 2008). KEG interacts with EDR1, which was shown to localize to the ER. EDR1 is recruited by KEG to the TGN/EE when coexpressed ( $\mathrm{Gu}$ and Innes, 2011). Another example is the previously mentioned $\mathrm{ARC1}$, shown to interact with Exo70A1, a subunit of the exocyst complex. Coexpression of Exo70A1 with ARC1 resulted in their relocalization from the cytosol to punctate structures (Samuel et al., 2009). Similarly, SYMRK relocalizes from the PM to punctate structures in the cytosol in the presence of SINA4 (Den Herder et al., 2012). However, whether target ubiquitination is required for the relocalization, still needs to be shown. Further work is necessary to resolve the dynamic interactions and modifications occurring between regulatory ligases and immune receptor kinases.

\section{CONCLUSIONS AND PERSPECTIVES}

Surfacing data showing the manifold and central functions of ubiquitination in vesicle trafficking represent a preliminary confirmation in plants of long standing paradigms in yeast and animal cells. However, the general scarcity of ubiquitination 
targets still obstructs insight into the cellular processes that are being regulated. Furthermore, it is necessary to discriminate between the different types of ubiquitination, since these mediate distinct fates of the tagged proteins. The importance of this aspect becomes apparent if one considers that ubiquitin is a common denominator involved in targeting of substrates to all three major protein degradation pathways in mammalian cells: the proteasome, the lysosome, and the autophagosome. In plants, most attention has been focused on the role of ubiquitination in

\section{REFERENCES}

Albrecht, C., Boutrot, F., Segonzac, C., Schwessinger, B., Gimenez-Ibanez, S., Chinchilla, D., et al. (2011). Brassinosteroids inhibit pathogenassociated molecular patterntriggered immune signaling independent of the receptor kinase BAK1. Proc. Natl. Acad. Sci. U.S.A. 109, 303-308.

Azevedo, C., Sadanandom, A., Kitagawa, K., Freialdenhoven, A., Shirasu, K., and Schulze-Lefert, P. (2002). The RAR1 interactor SGT1, an essential component of $\mathrm{R}$ gene-triggered disease resistance. Science 295, 2073 2076.

Barberon, M., Zelazny, E., Robert, S., Conejero, G., Curie, C., Friml, J., et al. (2011). Monoubiquitin-dependent endocytosis of the iron-regulated transporter 1 (IRT1) transporter controls iron uptake in plants. Proc. Natl. Acad. Sci. U.S.A. 108, E450-E458.

Belkhadir, Y., Jaillais, Y., Epple, P., Balsemao-Pires, E., Dangl, J. L., and Chory, J. (2011). Brassinosteroids modulate the efficiency of plant immune responses to microbeassociated molecular patterns. Proc. Natl. Acad. Sci. U.S.A. 109, 297-302.

Bent, A. F., and Mackey, D. (2007). Elicitors, effectors, and R genes: the new paradigm and a lifetime supply of questions. Annu. Rev. Phytopathol. 45, 399-436.

Cheng, Y. T., Li, Y., Huang, S., Huang, Y., Dong, X., Zhang, Y., et al. (2011). Stability of plant immune-receptor resistance proteins is controlled by SKP1Cullin1-F-box (SCF)-mediated protein degradation. Proc. Natl. Acad. Sci. U.S.A. 108, 14694-14699.

Chinchilla, D., Zipfel, C., Robatzek, S., Kemmerling, B., Nurnberger, T., Jones, J. D., et al. (2007). A flagellin-induced complex of the receptor FLS2 and BAK1 initiates plant defence. Nature 448, 497-500.

Chuang, T. H., and Ulevitch, R. J. (2004). Triad3A, an E3 ubiquitinprotein ligase regulating Toll-like receptors. Nat. Immunol. 5, 495-502.
Cooley, M. B., Pathirana, S., Wu, H. J., Kachroo, P., and Klessig, D. F. (2000). Members of the Arabidopsis HRT/RPP8 family of resistance genes confer resistance to both viral and oomycete pathogens. Plant Cell 12, 663-676.

Cui, F., Liu, L., Zhao, Q., Zhang, Z., Li, Q., Lin, B., et al. (2012). Arabidopsis ubiquitin conjugase UBC32 is an ERAD component that functions in brassinosteroid-mediated salt stress tolerance. Plant Cell 24, 233-244.

Den Herder, G., Yoshida, S., AntolinLlovera, M., Ried, M. K., and Parniske, M. (2012). Lotus japonicus E3 Ligase SEVEN IN ABSENTIA4 destabilizes the symbiosis receptorlike kinase SYMRK and negatively regulates rhizobial infection. Plant Cell 24, 1691-1707.

Gimenez-Ibanez, S., Hann, D. R., Ntoukakis, V., Petutschnig, E., Lipka, V., and Rathjen, J. P. (2009). AvrPtoB targets the LysM receptor kinase CERK1 to promote bacterial virulence on plants. Curr. Biol. 19, 423-429.

Göhre, V., Spallek, T., Haweker, H., Mersmann, S., Mentzel, T., Boller, T., etal. (2008). Plant patternrecognition receptor FLS2 is directed for degradation by the bacterial ubiquitin ligase AvrPtoB. Curr. Biol. 18, 1824-1832.

Gou, M., Su, N., Zheng, J., Huai, J., Wu, G., Zhao, J., et al. (2009). An F-box gene, CPR30, functions as a negative regulator of the defense response in Arabidopsis. Plant J. 60, 757-770.

Gou, M., Shi, Z., Zhu, Y., Bao, Z., Wang, G., and Hua, J. (2012). The F-box protein CPR1/CPR30 negatively regulates R protein $\mathrm{SNCl}$ accumulation. Plant J. 69, 411-420.

Gu, T., Mazzurco, M., Sulaman, W., Matias, D. D., and Goring, D. R. (1998). Binding of an arm repeat protein to the kinase domain of the S-locus receptor kinase. Proc. Natl. Acad. Sci. U.S.A. 95, 382-387.

$\mathrm{Gu}$, Y., and Innes, R. W. (2011). The KEEP ON GOING protein of Arabidopsis recruits the ENHANCED DISEASE RESISTANCE1 protein to trans-Golgi network/early endosome

mediating the turn-over of modified proteins by the proteasome, while relatively little is known about its role in directing proteins into the vacuole or autophagocytosis. However, at this point, the major challenge continues to be the identification of ligase targets.

\section{ACKNOWLEDGMENTS}

Work in the authors' laboratory is supported by the Leibniz Society and the Priority Program 1212 from the German Research Foundation (DFG).

vesicles. Plant Physiol. 155, $1827-$ 1838.

Haglund, K., Sigismund, S., Polo, S., Szymkiewicz, I., Di Fiore, P. P., and Dikic, I. (2003). Multiple monoubiq uitination of RTKs is sufficient for their endocytosis and degradation. Nat. Cell. Biol. 5, 461-466.

Herberth, S., Shahriari, M., Bruderek, M., Hessner, F., Muller, B., Hulskamp, M., et al. (2012). Artificial ubiquitylation is sufficient for sorting of a plasma membrane ATPase to the vacuolar lumen of Arabidopsis cells. Planta 236, 63-77.

Hicke, L. (2001). Protein regulation by monoubiquitin. Nat. Rev. Mol. Cell. Biol. 2, 195-201.

Hong, Z., Jin, H., Tzfira, T., and Li, J. (2008). Multiple mechanismmediated retention of a defective brassinosteroid receptor in the endoplasmic reticulum of Arabidopsis. Plant Cell 20, 3418-3429.

Husebye, H., Halaas, O., Stenmark, H., Tunheim, G., Sandanger, O., Bogen, B., et al. (2006). Endocytic pathways regulate Toll-like receptor 4 signaling and link innate and adaptive immunity. EMBO J. 25, 683-692.

Ikeda, F., and Dikic, I. (2008). Atypical ubiquitin chains: new molecular signals. 'Protein Modifications: Beyond the Usual Suspects' review series. EMBO Rep. 9, 536-542.

Isono, E., Katsiarimpa, A., Muller, I. K., Anzenberger, F., Stierhof, Y. D., Geldner, N., et al. (2010). The deubiquitinating enzyme AMSH3 is required for intracellular trafficking and vacuole biogenesis in Arabidopsis thaliana. Plant Cell 22, 1826-1837.

Janjusevic, R., Abramovitch, R. B., Martin, G. B., and Stebbins, C. E. (2006). A bacterial inhibitor of host programmed cell death defenses is an E3 ubiquitin ligase. Science 311, 222-226.

Jeong, R. D., Chandra-Shekara, A. C., Barman, S. R., Navarre, D., Klessig, D. F., Kachroo, A., et al. (2010). Cryptochrome 2 and phototropin 2 regulate resistance protein-mediated viral defense by negatively regulating an E3 ubiquitin ligase. Proc. Natl. Acad. Sci. U.S.A. 107, 13538-13543.
Jin, H., Yan, Z., Nam, K. H., and Li, J. (2007). Allele-specific suppression of a defective brassinosteroid receptor reveals a physiological role of UGGT in ER quality control. Mol. Cell 26, 821-830.

Jones, J. D., and Dangl, J. L. (2006). The plant immune system. Nature 444, 323-329.

Kasai, K., Takano, J., Miwa, K., Toyoda, A., and Fujiwara, T. (2011). High boron-induced ubiquitination regulates vacuolar sorting of the BOR1 borate transporter in Arabidopsis thaliana. J. Biol. Chem. 286, 6175-6183.

Katsiarimpa, A., Anzenberger, F., Schlager, N., Neubert, S., Hauser, M. T., Schwechheimer, C., et al. (2011). The Arabidopsis deubiquitinating enzyme AMSH3 interacts with ESCRT-III subunits and regulates their localization. Plant Cell 23, 3026-3040.

Kim, M., Cho, H. S., Kim, D. M., Lee, J. H., and Pai, H. S. (2003). CHRK1, a chitinase-related receptor-like kinase, interacts with NtPUB4, an armadillo repeat protein, in tobacco. Biochim. Biophys. Acta 1651, 50-59.

Li, W., Ahn, I. P., Ning, Y., Park, C. H., Zeng, L., Whitehill, J., et al. (2012). The U-box/ARM E3 ligase PUB13 regulates cell death, defense and flowering time in Arabidopsis. Plant Physiol. 159, 239-250.

Liu, Y., Schiff, M., Serino, G., Deng, X. W., and Dinesh-Kumar, S. P. (2002). Role of SCF ubiquitin-ligase and the COP9 signalosome in the $\mathrm{N}$ gene-mediated resistance response to Tobacco mosaic virus. Plant Cell 14, 1483-1496.

Lu, D., Lin, W., Gao, X., Wu, S., Cheng, C., Avila, J., et al. (2011). Direct ubiquitination of pattern recognition receptor FLS2 attenuates plant innate immunity. Science 332, 1439-1442.

Mbengue, M., Camut, S., de CarvalhoNiebel, F., Deslandes, L., Froidure, S., Klaus-Heisen, D., et al. (2010). The Medicago truncatula E3 ubiquitin ligase PUB1 interacts with the LYK3 symbiotic receptor and negatively regulates infection and nodulation. Plant Cell 22, 3474-3488. 
Monaghan, J., and Zipfel, C. (2012). Plant pattern recognition receptor complexes at the plasma membrane. Curr. Opin. Plant Biol. 15, 349-357.

Müller, J., Piffanelli, P., Devoto, A., Miklis, M., Elliott, C., Ortmann, B., et al. (2005). Conserved ERAD-like quality control of a plant polytopic membrane protein. Plant Cell 17, 149-163.

Nekrasov, V., Li, J., Batoux, M., Roux, M., Chu, Z. H., Lacombe, S., et al. (2009). Control of the patternrecognition receptor EFR by an ER protein complex in plant immunity. EMBO J. 28, 3428-3438.

Raiborg, C., Rusten, T. E., and Stenmark, H. (2003). Protein sorting into multivesicular endosomes. Curr. Opin. Cell Biol. 15, 446-455.

Robatzek, S., Chinchilla, D., and Boller, T. (2006). Ligand-induced endocytosis of the pattern recognition receptor FLS2 in Arabidopsis. Genes Dev. 20, 537-542.

Saijo, Y., Tintor, N., Lu, X., Rauf, P., Pajerowska-Mukhtar, K., Haweker, H., et al. (2009). Receptor quality control in the endoplasmic reticulum for plant innate immunity. $E M B O J$. 28, 3439-3449.

Samuel, M. A., Mudgil, Y., Salt, J. N., Delmas, F., Ramachandran, S. Chilelli, A., et al. (2008). Interactions between the S-domain receptor kinases and AtPUB-ARM E3 ubiquitin ligases suggest a conserved signaling pathway in Arabidopsis. Plant Physiol. 147, 2084-2095.
Samuel, M. A., Chong, Y. T., Haasen, K. E., Aldea-Brydges, M. G., Stone, S. L., and Goring, D. R. (2009). Cellular pathways regulating responses to compatible and self-incompatible pollen in Brassica and Arabidopsis stigmas intersect at Exo70A1, a putative component of the exocyst complex. Plant Cell 21, 2655-2671.

Scita, G., and Di Fiore, P. P. (2010). The endocytic matrix. Nature 463, 464-473.

Shirasu, K. (2009). The HSP90-SGT1 chaperone complex for NLR immune sensors. Annu. Rev. Plant Biol. 60 139-164.

Smith, M. H., Ploegh, H. L., and Weissman, J. S. (2011). Road to ruin: targeting proteins for degradation in the endoplasmic reticulum. Science 334, 1086-1090.

Sorkin, A., and von Zastrow, M. (2009). Endocytosis and signalling: intertwining molecular networks. Nat Rev. Mol. Cell Biol. 10, 609-622.

Stone, S. L., Anderson, E. M., Mullen, R. T., and Goring, D. R. (2003). ARC1 is an E3 ubiquitin ligase and promotes the ubiquitination of proteins during the rejection of self-incompatible Brassica pollen. Plant Cell 15, 885-898.

Stone, S. L., Arnoldo, M., and Goring, D. R. (1999). A breakdown of Brassica self-incompatibility in ARC1 antisense transgenic plants. Science 286, 1729-1731.

Su, W., Liu, Y., Xia, Y., Hong, Z., and Li, J. (2010). Conserved endoplasmic reticulum-associated degradation system to eliminate mutated receptor-like kinases in Arabidopsis. Proc. Natl. Acad. Sci. U.S.A. 108 870-875.

Trujillo, M., Ichimura, K., Casais, C. and Shirasu, K. (2008). Negative regulation of PAMP-triggered immunity by an E3 ubiquitin ligase triplet in Arabidopsis. Curr. Biol. 18, 13961401.

Vega-Sanchez, M. E., Zeng, L., Chen, S., Leung, H., and Wang, G. L. (2008) SPIN1, a K homology domain protein negatively regulated and ubiquitinated by the E3 ubiquitin ligase SPL11, is involved in flowering time control in rice. Plant Cell 20, 1456 1469.

Vierstra, R. D. (2009). The ubiquitin$26 \mathrm{~S}$ proteasome system at the nexus of plant biology. Nat. Rev. Mol. Cell Biol. 10, 385-397.

Wang, Y. S., Pi, L. Y., Chen, X., Chakrabarty, P. K., Jiang, J., De Leon, A. L., et al. (2006). Rice XA21 binding protein 3 is a ubiquitin ligase required for full Xa21-mediated disease resistance. Plant Cell 18, 3635-3646.

Wawrzynska, A., Christiansen, K. M., Lan, Y., Rodibaugh, N. L., and Innes, R. W. (2008). Powdery mildew resistance conferred by loss of the ENHANCED DISEASE RESISTANCE1 protein kinase is suppressed by a missense mutation in KEEP ON GOING, a regulator of abscisic acid signaling. Plant Physiol. 148, 1510-1522.
Zeng, L. R., Qu, S., Bordeos, A. Yang, C., Baraoidan, M., Yan, H., et al. (2004). Spotted leaf11, a negative regulator of plant cell death and defense, encodes a U-box/armadillo repeat protein endowed with E3 ubiquitin ligase activity. Plant Cell 16, 2795-2808.

Conflict of Interest Statement: The authors declare that the research was conducted in the absence of any commercial or financial relationships that could be construed as a potential conflict of interest.

Received: 28 June 2012; paper pending published: 30 July 2012; accepted: 09 October 2012; published online: 24 October 2012

Citation: Furlan G, Klinkenberg J and Trujillo $M$ (2012) Regulation of plant immune receptors by ubiquitination. Front. Plant Sci. 3:238. doi: 10.3389/ fpls.2012.00238

This article was submitted to Frontiers in Plant Proteomics, a specialty of Frontiers in Plant Science.

Copyright (c) 2012 Furlan, Klinkenberg and Trujillo. This is an open-access article distributed under the terms of the Creative Commons Attribution License, which permits use, distribution and reproduction in other forums, provided the original authors and source are credited and subject to any copyright notices concerning any third-party graphics etc. 